International Journal of Distributed and Parallel Systems (IJDPS) Vol.3, No.5, September 2012

\title{
EVALUATION OF PARAMETERS FOR IMPROVING HANDoff PERFormance in Mobile WimaX NETWORKS
}

\author{
Sharmistha Khan ${ }^{1}$, Ji Hyun Lee ${ }^{2}$, Golam Rosul Khan ${ }^{1}$ \\ ${ }^{1}$ Department of Electrical and Computer Engineering, Prairie View A\&M University, \\ Prairie View, TX \\ skhan2@student. pvamu . edu; gkhanestudent.pvamu . edu \\ ${ }^{2}$ Department of Electrical Engineering, Tuskegee University, Tuskegee Institute, AL \\ jhlee@mytu.tuskegee.edu
}

\begin{abstract}
Mobile WiMAX is the latest technology that promises broadband wireless access over long distance. In order to support mobility and continuous network connectivity in mobile WiMAX, it is necessary to provide handoff. Handoff is an essential process in wireless networks to guarantee continuous and effective services. This paper focuses on the analysis of the performance of the handoff process in Mobile WiMAX. This paper aims to find out the factors/parameters of the WiMAX module that affect handoff performance the most, such as handoff duration of less than $50 \mathrm{~ms}$ and mobility speed up to 120km/hour. Simulation results show that some of the parameters of WiMAX do not have any influence on handoff latency, while others have great impact towards achieving shorter handoff duration time. The results also show that handoff times could vary for different speeds of the Mobile Station (MS).
\end{abstract}

\section{KEYWORDS}

Handoff, Mobile WiMAX.

\section{INTRODUCTION}

In early 2001, the WiMAX Forum developed the most modern wireless technology named WiMAX, which is a telecommunications protocol that provides fixed and fully mobile Internet access. There are many positive aspects of this technology; one of the most important is the support of a large coverage area. WiMAX provides the support of wireless connectivity with a minimum range of 30 miles. WiMAX technology also offers high speed broadband access to mobile internet which transfers data, voice, and video. In WiMAX, when a user uses a $20 \mathrm{MHz}$ data rate the bandwidth for this data rate can be up to $75 \mathrm{Mbps}$. Whereas WiFi offers a short range of data transfer with a maximum bandwidth of $54 \mathrm{Mbps}$ [1] [2].

The IEEE 802.16 standard forms the basis of WiMAX technology. The WiMAX Forum gradually improves the functionality and approves different generations for this standard. Usually these standards differ in two different forms generally known as "802.16d" or "Fixed WiMAX" and "802.16e" or "Mobile WiMAX". The 802.16d-2004 standard has no support for mobility. Mobility support is when a user is in motion or in a vehicle and can easily access the wireless network. To solve the problem of mobility the IEEE 802.16e-2005 standard was 
International Journal of Distributed and Parallel Systems (IJDPS) Vol.3, No.5, September 2012

published and had full support for mobility. Since this standard introduced the support for mobility it is known as "Mobile WiMAX" [2].

In Mobile WiMAX networks when a Mobile Node (MN) changes its location the MN moves the point of attachment to the network. In this situation it is essential to provide continuous network connectivity to satisfy high levels of mobile service quality. Here the major issue concerning implementation of Mobile WiMAX is providing effective handoff. Handoff is the process of changing a Mobile Station's (MS's) network connectivity from one Base Station (BS) to another BS. Providing the support for ongoing video call, or Voice over IP (VoIP), conversations for mobile users makes it necessary to make the handoff process as fast as possible. Therefore, in order to decrease handoff interruption time and unnecessary call drops it is important to implement different handoff optimization methods. Providing different Quality of Service (QoS) support such as high speed data transmission, low handoff latency, a reduced amount of packet loss in Mobile WiMAX network, and various handoff enhancement approaches have been proposed.

For the past few years of Mobile WiMAX technology many researchers have explained the handoff process in Mobile WiMAX along with different handoff techniques. Some proposed effective algorithms for improving handoff methods; while others proposed vertical handoff schemes with the aim of reducing handoff signaling overhead on the wireless backbone and providing a low handoff delay to mobile nodes [3]. Other researchers have introduced Secure Internet Protocol (IPSec) based end-to-end securing solutions for real-time services, which provide a secure and fast seamless handoff solution while preserving the QoS and security when moving between heterogeneous access networks [4]. Again, various fast handoff techniques have also been suggested in literature to meet QoS requirements. In general, we found that different researchers worked on different aspects but with the same general motto, to improve handoff performance in Mobile WiMAX. All of the handoff techniques were implemented in such a way that they always supported short interruption time, low handoff latency, high speed, and were cost effective. Still, a great deal of research is being conducted in order to determine a more efficient handoff process to reach the desired performance. If we want to improve the performance of the handoff methods we need to first analyze the present condition of the performance of the implemented handoff processes in Mobile WiMAX. The performance of the handoff processes are regulated by the parameters of the WiMAX module. Therefore, we need to adjust those parameters in order to increase handoff performance by achieving shorter handoff duration time. The velocity of the MS may also have some impact on the performance of the handoff process. Therefore, our main focus is to analyze the performance of the handoff methods in Mobile WiMAX by investigating different performance related parameters with varied MS velocity.

This paper is organized as follows. After the introduction, the handoff in Mobile WiMAX is described in Section 2. Simulation results are presented and discussed in Section 3. Based on the simulation results, conclusions are given in Section 4.

\section{HANDOFF IN MOBILE WIMAX}

Mobility is one of the most important features in wireless cellular communication. When a mobile node changes its location from one place to another, to provide mobility support, the minimum requirements are reliable, efficient, and continuous data transmission across the air interface. Generally, this continuous data transmission service can be achieved by implementing 
International Journal of Distributed and Parallel Systems (IJDPS) Vol.3, No.5, September 2012

a handoff from one cell to another. Handoff is a process with the intention of changing the network access point of a mobile node without any data loss or disturbance of the current connection while a call is in progress. Therefore, in mobile WiMAX, the basic meaning of handoff process is to provide uninterrupted connectivity when a MS transfers from the airinterface of one BS to the air-interface of another BS. Though handoff is usually understood as a change of physical connection of a serving BS, it does not mean that the BS must be changed. Sometimes handoff can occurr within different channels, this means changing from one frequency to another while the serving BS remains the same. In mobile WiMAX, it is called intra-cell handoff while the second option is called inter cell handoff. Again, in some other cases, handoff can occur within different network technologies. When handoff occurs within a single network it is known as horizontal handoff, while handoff between different networks is known as vertical handoff. To implement a handoff process requires at least two BSs, the currently serving BS and target BS, and a MS within the range of the two BSs [5] [6].

\subsection{Reasons of Handoff}

Telecommunication reasons for conducting handoff can vary. Here we have mentioned some of these cases.

- When the cellular phone is moving away from the area covered by the serving BS, the device eventually goes outside the range of the serving BS. So in order to avoid call termination, the call needs to be transferred to an area covered by another BS [5].

- When the signal strength is not enough to maintain a proper call at the edge of a serving cell, the call needs to be transferred to another cell [6].

- When the capacity for connecting new calls of a cell in a BS becomes full or more traffic is pending, capacity must be made available for users who can only be connected on that cell. Therefore, the existing calls or the new calls from a phone that is located in an area that is overlapped by both cells can be transferred from the first cell to the second cell [5] [6].

- In non-CDMA networks several phones use different cells but the same channel. As a channel is being used by several phones, disturbing co-channel interference comes from another phone. Therefore, in order to avoid interference, the call is transferred to a different channel in the same cell or to a different channel in another cell [5].

- In vertical handoff, a faster network is occasionally available. So the phone changes its network to the cheaper one [6].

\subsection{Types of Handoff}

Handoff is divided roughly into two broad categories: hard and soft handoffs with different variants based on the technology used. They are also known as "break before make" and "make before break" handoffs. Generally, in hard handoffs old connections are broken before new connections are created; in soft handoffs, both existing and new connections are used during the handoff process. IEEE 802.16e divided the handoff process into three major classifications Hard Handoff (HHO), Macro Diversity Handoff (MDHO), and Fast Base Station Switching (FBSS), while HHO is mandatory in WiMAX systems, the other types of handoff are optional [7]. 
The HO procedures can be divided into two major phases known as network topology acquisition parts and the actual $\mathrm{HO}$ process. The network topology acquisition phase can be divided into 3 sub procedures: network topology advertisement, MS scanning of neighbor BSs, and association procedure. These three sub-procedures are executed before $\mathrm{HO}$ through the backbone network. Again, the actual HO phase can be divided into other sub phases: cell selection, handoff decision and initiation, synchronizing with new downlink and obtain parameters, obtaining uplink parameters, ranging and uplinking parameter adjustment, MS reauthorization, re-registering, and termination with the serving BS [6]. All of these sub-phases are executed during HO.

\section{SIMULATION AND RESULTS}

The main goal of these simulations was to investigate the impact of different parameters of the WiMAX module during handoff as well as examine the properties of Mobile WiMAX. A simple and basic scheme was designed in order to keep the simulation process simple and trouble-free. To implement handoff in Mobile WiMAX we have used the Network Simulator 2 (NS-2 Version 2.29), which forms a basis for the simulations. Additionally, in order to support Mobile WiMAX, we have to use two other particular modules known as WiMAX and Mobility module from the NIST project [8]. Again, the additional modules also lack some functionality to support the implementation of Mobile WiMAX. Therefore, we tried to get the results and measurements as accurately as possible from the simulation according to the simulator's perspective.

The simulation scenario consists of three 802.16e BSs (BS0, BS1, BS2) and one MS; where the BSs are aligned in a line and the MS travels through the coverage areas of these three BSs. The BSs are located in such a way that there will be some overlap within the coverage areas of the neighboring BSs. We selected constant values for some of the elements such as cell size, transmission power of BSs, and the route of the MS. Our main concern was to adjust the parameters of the WiMAX module to achieve faster handoff times in the NS-2. We simulated the whole scenario with speeds of 10 meters/second to 40 meters/second with 1 meter/second steps. The first handoff will occur when the MS reaches the edge of the coverage area of BS0 and it needs to change its connection access point from BS0 to BS1 for continuous service. The second handoff will occur when the MS reaches edge of the coverage area of the BS1 where it needs to once again change its connection access point from BS1 to BS2.

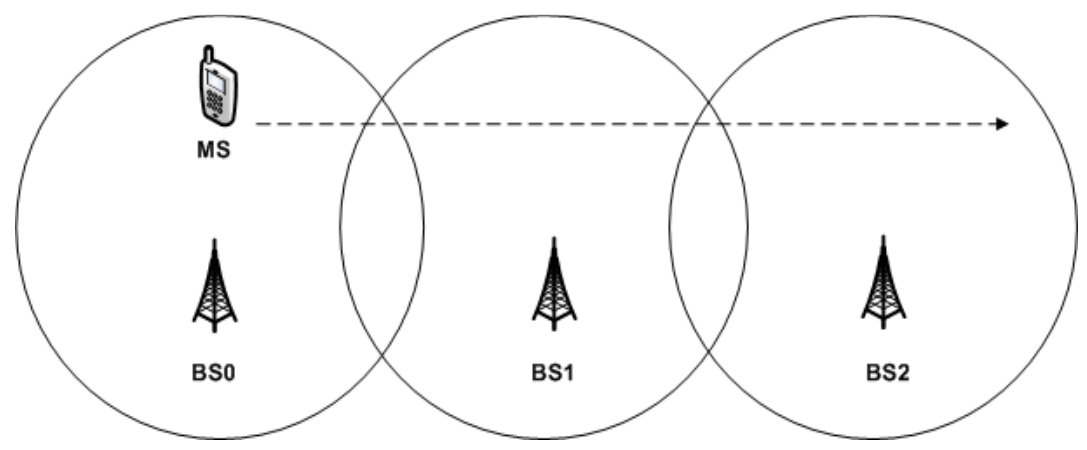

Figure 1. Simulation Scenario 
International Journal of Distributed and Parallel Systems (IJDPS) Vol.3, No.5, September 2012

In simulation, our main goal was to find out the parameters that have the greatest impact on the performance of the handoff process in Mobile WiMAX. According to the scenario, we have measured the time for the two handoffs.

\subsection{Adjusted Parameters}

In order to achieve less handoff time we have adjusted the list of parameters of the WiMAX module. When we changed the value of a parameter we ran the simulation and measured the handoff time for each adjustment. We changed the values of some parameters from the example file of the WiMAX module which is programmed with predefined values. Then we adjusted the rest of the parameters that are offered by the MAC/ 802.16 in NS-2 and have some impact on the handoff time. Some of the parameters were set as their default value in the simulation code. When we completed the adjustment successfully, we found that some of the parameters did not have any impact on handoff latency, while other parameters had a great influence. We will discuss these parameters in brief description in the simulation result section.

\subsection{Constant Parameters}

As the implementation process of handoff in Mobile WiMAX is quite complex we want to keep the simulation process simple. Therefore, we have used constant values for some of the parameters that are related to the configuration of the BSs such as the BS coverage area, transmission power, and operating frequency. By keeping these parameters constant we could adjust other parameters to achieve less handoff duration time for both handoffs. For each adjustment we used the same simulation scenario, where the number of BSs, location of the BSs, and the route of the MS were mentioned. The bit rate for transmitting data was also kept constant around 1.4 Mbps. From literature, we found that to send a MPEG-1 file a data transmission bit rate of $1.5 \mathrm{Mbps}$ is required [9]. Comparing this value with the used data bit rate of this simulation can be considered as a moderate bit rate. The packet size and the duration time for sending a packet were also kept constant, 1600 bytes and $15 \mathrm{~ms}$ respectively.

\subsection{Simulation Result}

Our main focus of this simulation was to adjust the values of the different parameters with varied MS speed during the two handoffs. After completing the adjustment of all the parameters, we found that the handoff times were $36.49 \mathrm{~ms}$ for the first handoff and $37.76 \mathrm{~ms}$ for the second handoff, both of which are less than $50 \mathrm{~ms}$ which is the standard for the handoff latency defined by the WiMAX forum. The following section will discuss the influence of the parameters that we adjusted in the simulation, and later on we will discuss the impact of varied MS speeds on handoff times.

\subsubsection{Lgd_factor}

The Link Going Down Factor is one of the most important parameters of the WiMAX module, as it determines the sensitivity of detecting a falling link. We need to set this factor to generate a Link Going Down. When the received power of a signal is less than factor RXThresh, a trigger is generated to initiate scanning for neighbor BSs.

For a certain coverage area, at the edge, RXThresh value defines the limit of that area that means outside of that area the data packet will be discarded. The higher the factor the sooner the trigger is generated. If we set the factor value to 1 , it does not detect any falling link. The optimal factor value ranged from 1.3 to 1.6 as shown in Figure 2, so we chose the best value as 1.5. 


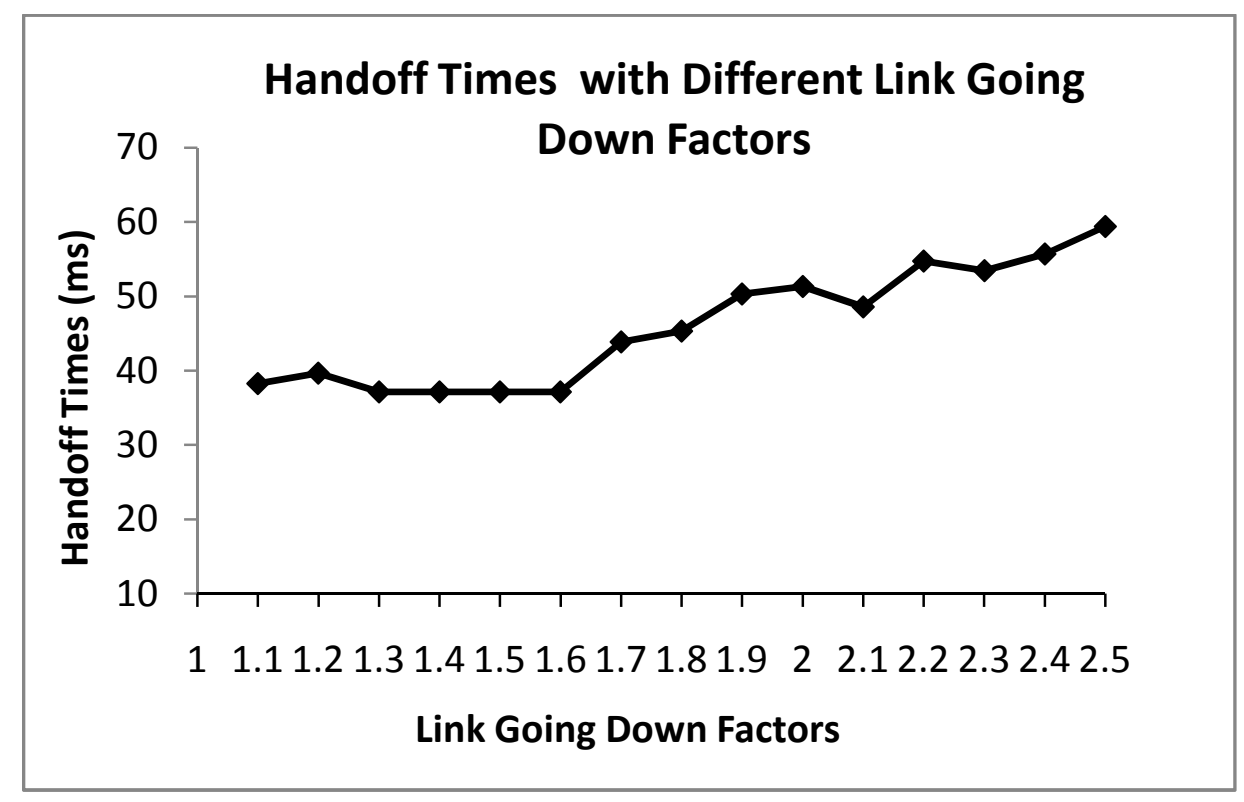

Figure 2. Handoff Times vs Link Going Down Factors

\subsubsection{Scan_iteration}

Scan iteration defines the requested number of iterating scanning interval by an MS, which means how many times the MS will complete the scanning procure. We have found that the more iteration time provided, the longer handoff duration time. We have seen from Figure 3 that the best value is two iterations.

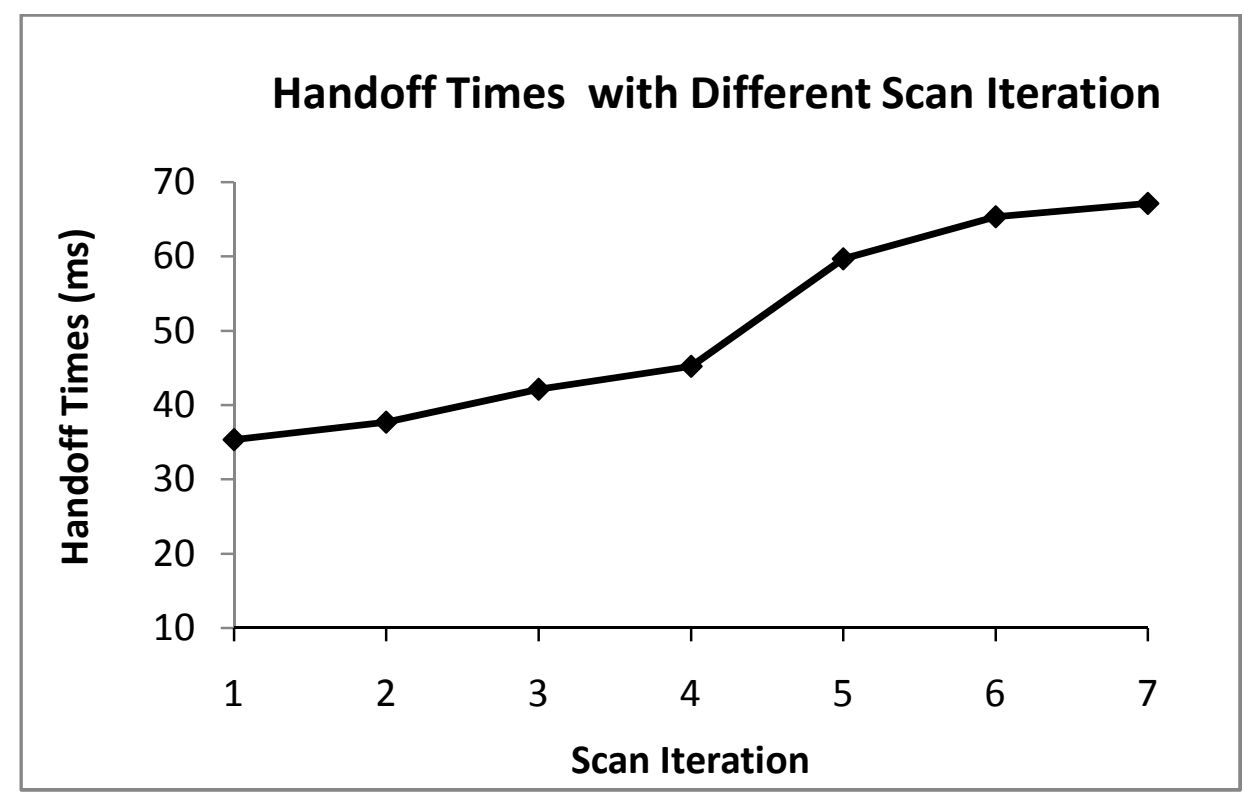

Figure 3. Handoff Times vs. Scan Iteration 


\subsubsection{Scan_duration}

Scan duration is another important parameter of the WiMAX module, which defines the length of the scanning period in frames. In order to make a successful scan, long duration time is desirable. On the other hand, short duration time for scanning also produces moderate elapsed time. We have found the best value is six frames with frame duration of $5 \mathrm{~ms}$.

\subsubsection{Interleaving_interval}

Interleaving interval is the parameter that defines the time duration between the normal operation and scanning periods of the MS in frames. We have seen from Figure 4 that if we set the value less than or equal to twenty frames it will affect the handoff time with a small variation. However, increasing the value to more than twenty frames causes longer handoff times. We got the best value at nine frames.

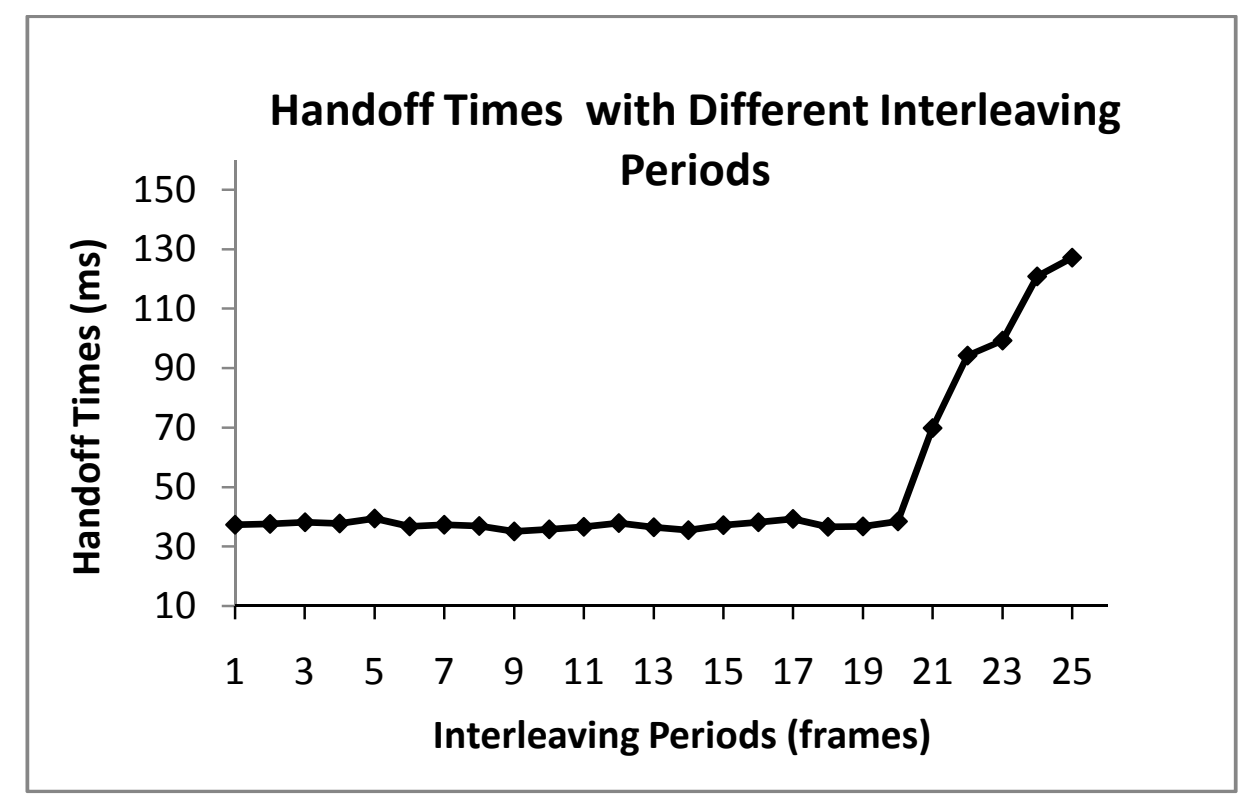

Figure 4. Handoff Times vs. Interleaving Periods

\subsubsection{T21_timeout}

T21 timeout is the parameter that defines the timeout value for the MS for searching the DLMAP message, which means the MS needs to find a DL-MAP message within this period. We found the same handoff time when the timeout was set between 5 to $27 \mathrm{~ms}$, as shown in Figure 5. However, if we increase $1 \mathrm{~ms}$ after $27 \mathrm{~ms}$ it increases the handoff time drastically. We set this timeout value to $20 \mathrm{~ms}$.

\subsubsection{Frame_duration}

Frame duration defines the length or the size of a frame. From literature, we have found that a suitable value for the frame duration is 4 or $5 \mathrm{~ms}$. We chose $5 \mathrm{~ms}$ for our simulation. 
International Journal of Distributed and Parallel Systems (IJDPS) Vol.3, No.5, September 2012

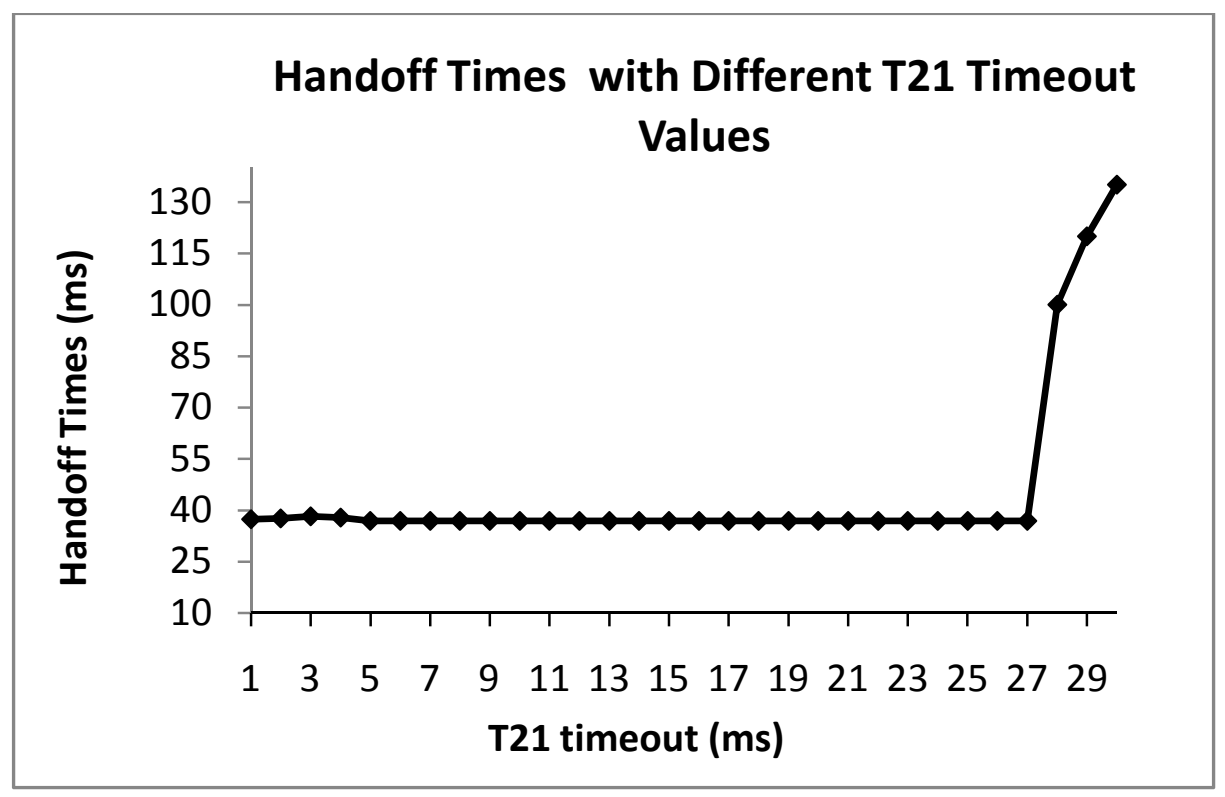

Figure 5. Handoff Times vs. T21 Timeout Values

\subsubsection{Lost_dlmap_interval}

Lost DL-MAP interval defines the timeout value for last reception of a DL-MAP message in the MS, which means that when a new DL-MAP message does not arrive to the MS within this time period the synchronization between the MS and BS will be interrupted.

From the simulation, we have investigated that a value of less than $9 \mathrm{~ms}$ causes a longer handoff time. Sometimes it even increases the handoff time by several seconds. We have seen from Figure 6 that the best result comes at $15 \mathrm{~ms}$.

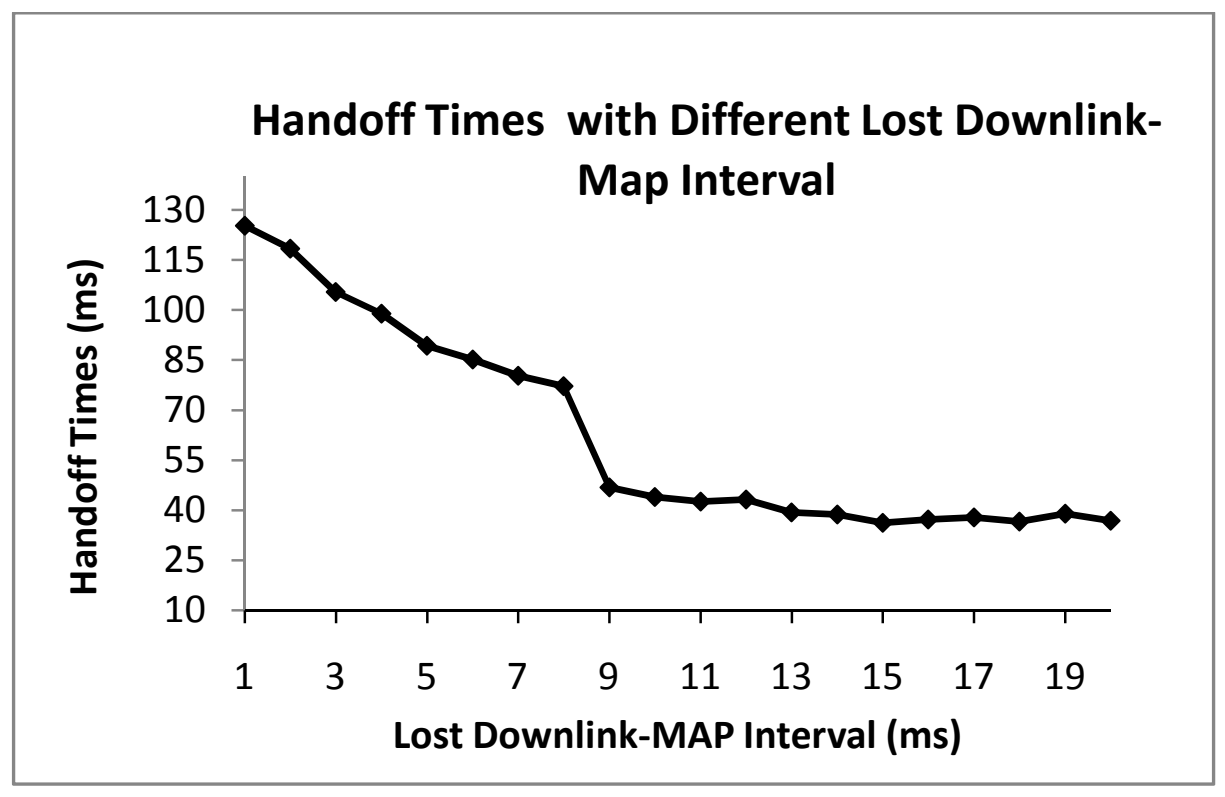

Figure 6. Handoff Times vs. Lost Downlink-MAP Interval 


\subsubsection{Client_timeout}

This is one kind of timer value that detects the range out of the MS. We have seen that smaller timer value is not efficient for a MS travelling within a large coverage area. On the other hand, greater timer values also cause longer delays. We found the best value to be $6 \mathrm{~ms}$.

\subsubsection{Lost_ulmap_interval}

The functionalities of this parameter are similar to the previous one, but it is used for UL-MAP message. From Figure 7, we have found that values less than $5 \mathrm{~ms}$ cause a longer delay in handoff. So, the preferable value during simulation is $15 \mathrm{~ms}$.

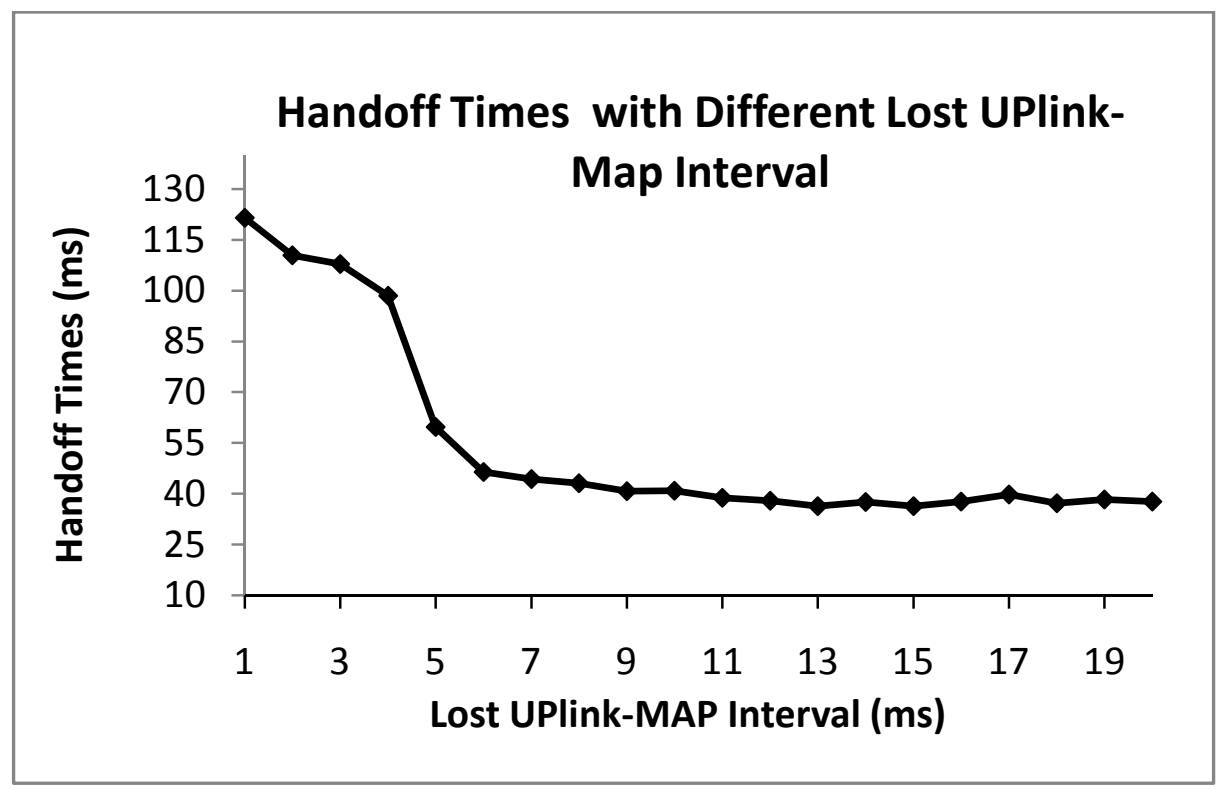

Figure 7. Handoff Times vs. Lost Uplink-MAP Interval

\subsubsection{Ranging_backoff_start}

Ranging backoff start is a parameter used during the ranging phase of the handoff. It defines the size for initial backoff window. We have found the same handoff time for a window size of 2 to 5 slots, which is shown in Figure 8.

If we increase the slot size to more than 5 it increases handoff time. On the other hand, we have also seen that a slot size of 1 also causes longer handoff time. Therefore, we have used 3 slots as a backoff window size for initial ranging. 
International Journal of Distributed and Parallel Systems (IJDPS) Vol.3, No.5, September 2012

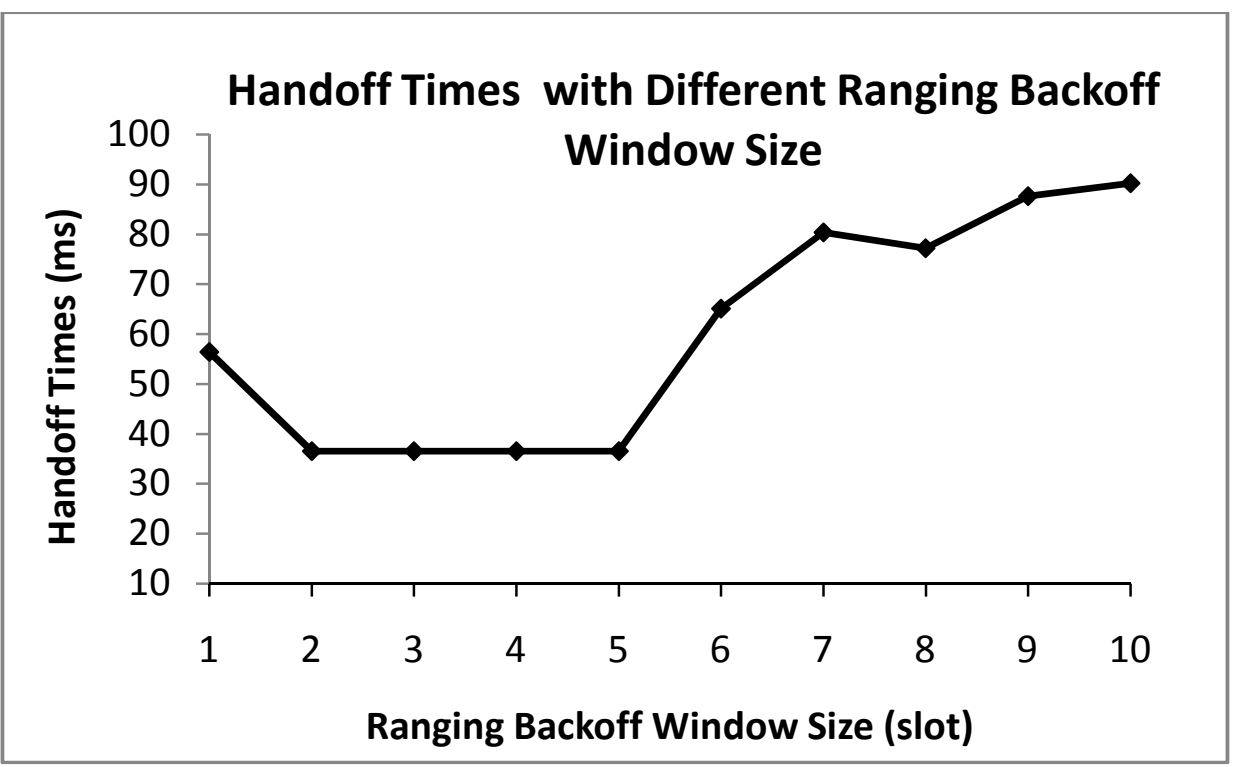

Figure 8. Handoff Times vs. Ranging Backoff Window Size

\subsubsection{MaxRADelay}

This parameter was used to define the maximum delay for sending a reply message to an RS. Larger values cause larger handoff delays. We have seen from Figure 9 that a value less than or equal to $6 \mathrm{~ms}$ achieves the best results. Therefore, we have selected the value for the maximum delay as $4 \mathrm{~ms}$.

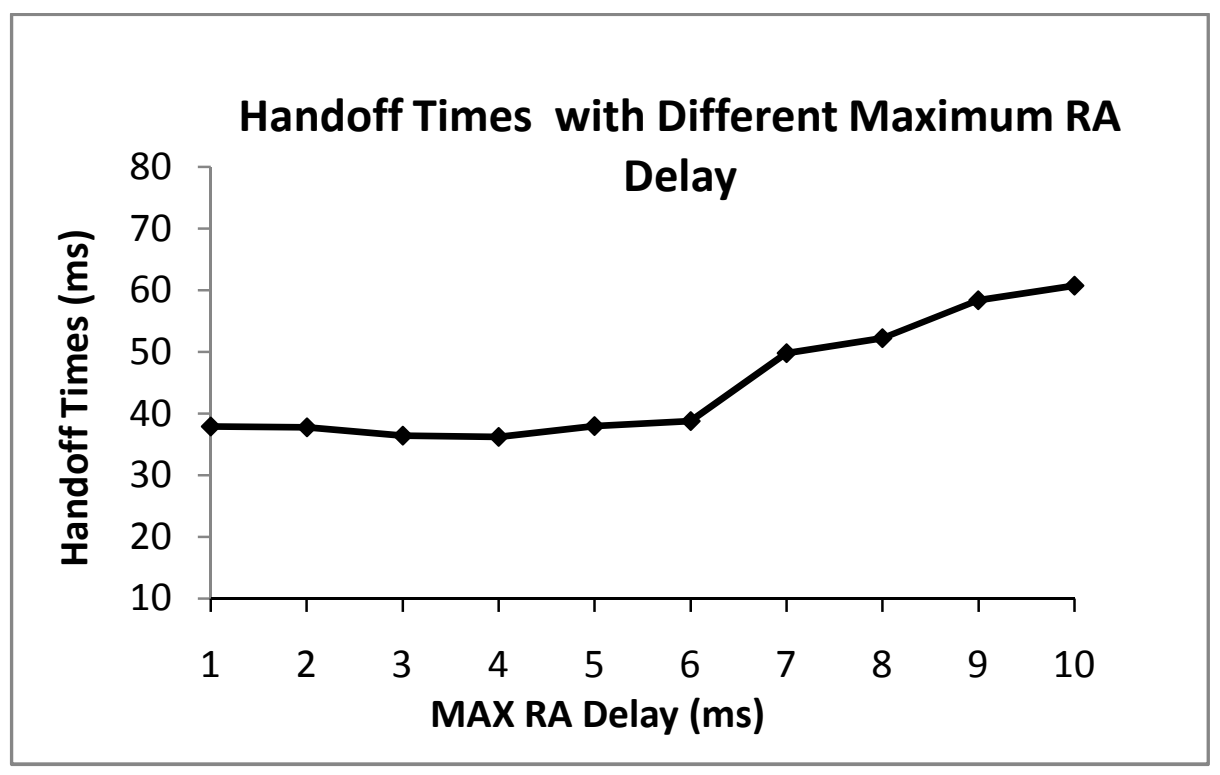

Figure 9. Handoff Times vs. Maximum RA Delay Values 


\subsubsection{MinDelayBetweenRA}

This parameter overrides the default value of the minimum interval time between two consecutive RAs and has a great impact on handoff time.

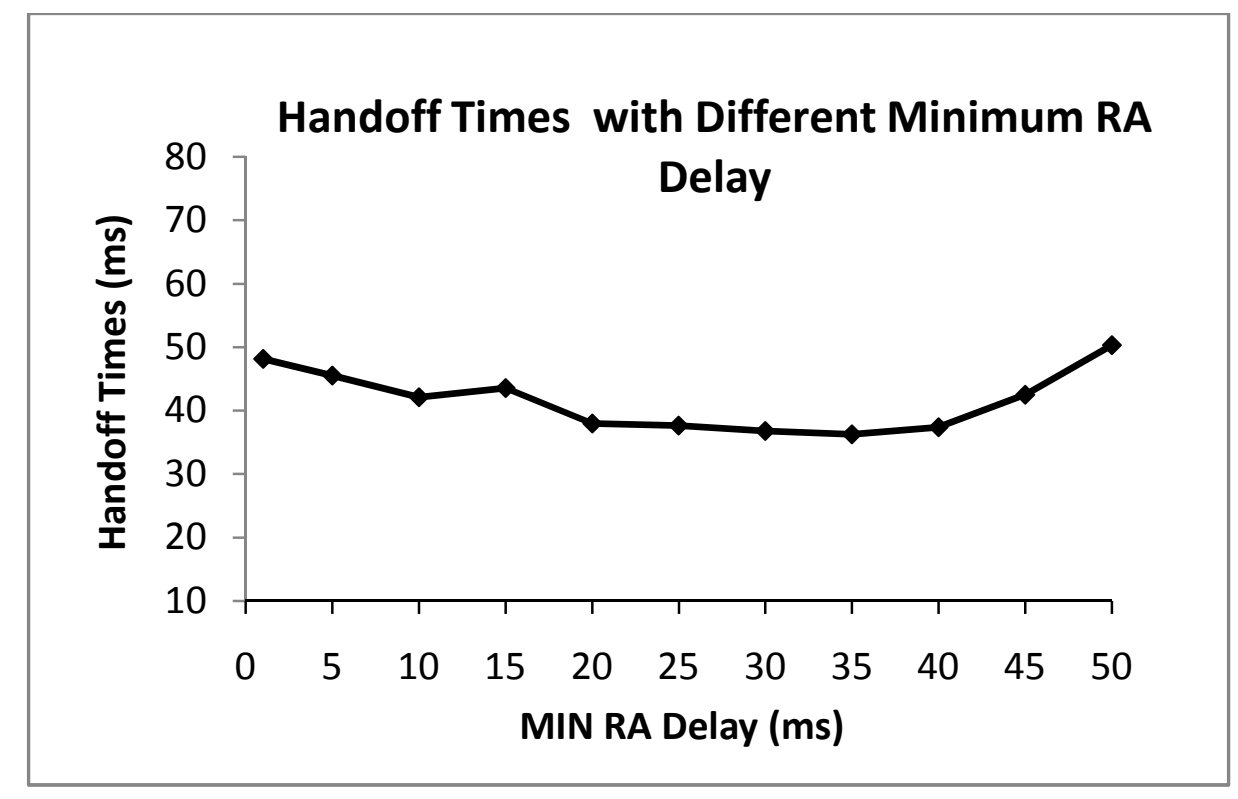

Figure 10. Handoff Times vs. Minimum RA Delay Values

From Figure 10 we have found that the best handoff time occurs when the delay was at a minimum of $35 \mathrm{~ms}$.

\subsubsection{Queue_length}

Queue length parameter is basically used during data transmission from the MS. It defines the length of the buffer for a sending packet. As the data rate we have used in the simulation is not very large, it does not require a large buffer. The data rate for packet size 2 was used in the simulation, which is enough to run the simulation. We found that if we chose a buffer size of 1 packet, some data fell apart during transmission. So the best value we found is 2 packets.

\subsubsection{T44_timeout}

This parameter defines the timeout value for a successful scanning request during the scanning phase. We have investigated from the simulation result that this value needs to be more than 5 ms. We chose the default value $10 \mathrm{~ms}$, which was defined in the WiMAX module.

\subsubsection{MinRtrAdvInterval}

This is one of the most important parameters of the ND module and defines the minimum interval between consecutive RAs. The large interval value causes longer handoff time. We have found that the best result comes with the interval period of zero. 


\subsubsection{MaxRtrAdvInterval}

This parameter is similar to the one used above but is used for maximum interval. We have found that the best result comes with the interval period of $10 \mathrm{~ms}$.

\subsection{Handoff Times with Varied MS Velocity}

Investigating the impact of different velocities of the MS is one of our main concerns of this research. During the adjustment of the different parameters, we also varied the velocity of the MS. We have done simulation by changing the speeds of the MS from $10 \mathrm{~m} / \mathrm{s}$ to $40 \mathrm{~m} / \mathrm{s}$ with a 1 $\mathrm{m} / \mathrm{s}$ step. We chose the highest speed $40 \mathrm{~m} / \mathrm{s}$ as it equals $144 \mathrm{~km} / \mathrm{h}$, which is well above to the standard limit $(100 \mathrm{~km} / \mathrm{h})$ for a seamless handoff [7].

When we adjusted the parameters we varied the speed and investigated the results of the handoff times. The impacts of the different velocities of the MS on handoff times are presented in Figure 11 and Figure 12. We found that for the MS speed of $10 \mathrm{~m} / \mathrm{s}$ to $19 \mathrm{~m} / \mathrm{s}$, the handoff times varied consistently by a few milliseconds within the range of $35 \mathrm{~ms}$ to $45 \mathrm{~ms}$, which are less than 50 $\mathrm{ms}$. After $20 \mathrm{~m} / \mathrm{s}$ MS speed, the handoff times increased inconsistently and went up to approximately $150 \mathrm{~ms}$, much larger than the latency limit. But this result can differ for real time traffic because here the MS speeds were controlled smoothly by the simulator. On the other hand, due to the lack of the simulator, we could not implement some of the major features of Mobile WiMAX like QoS, authentication, and service flow. Therefore, the results can also vary for those missing features.

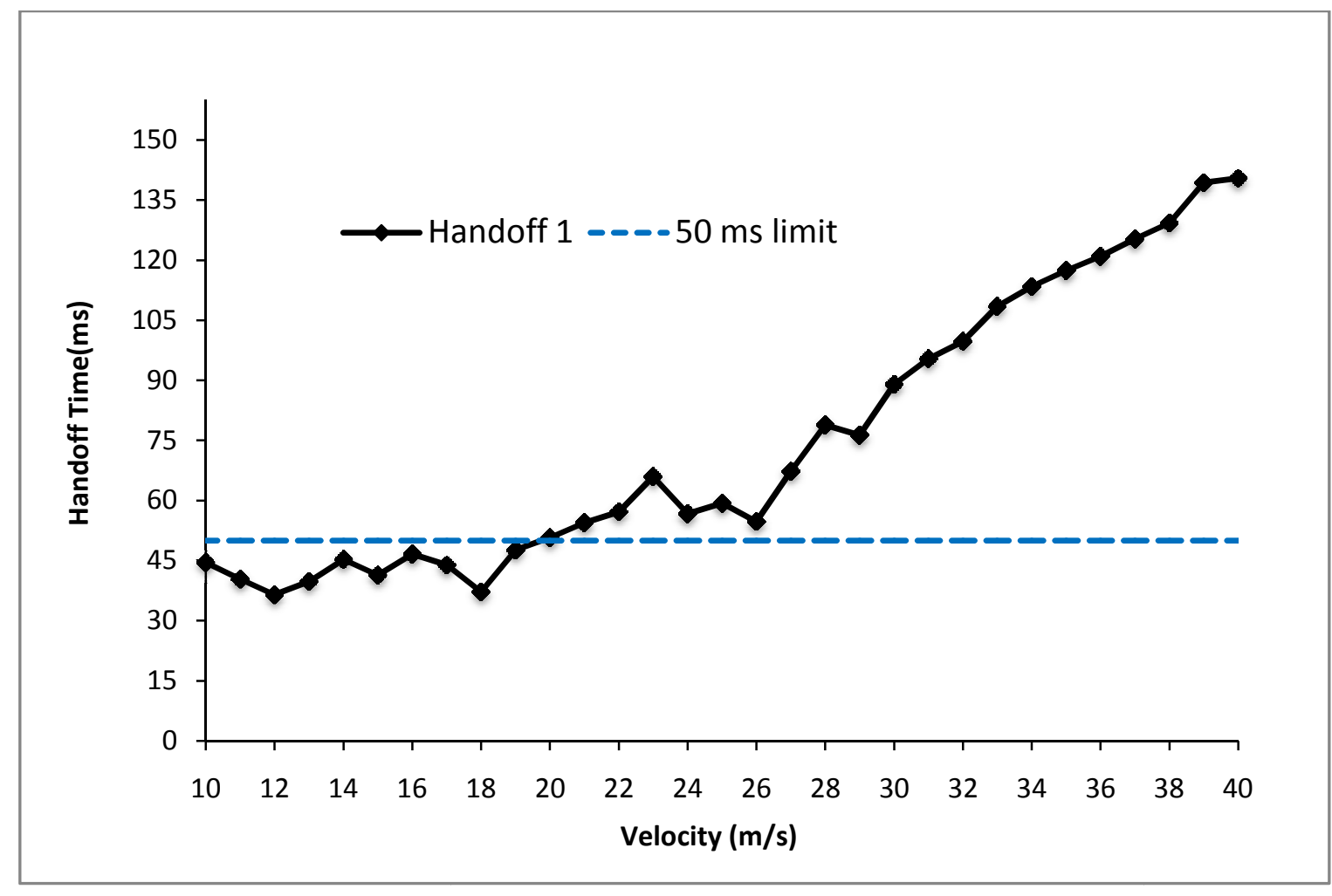

Figure 11. Simulated Handoff Times with Varied MS Speeds for First Handoff 
International Journal of Distributed and Parallel Systems (IJDPS) Vol.3, No.5, September 2012

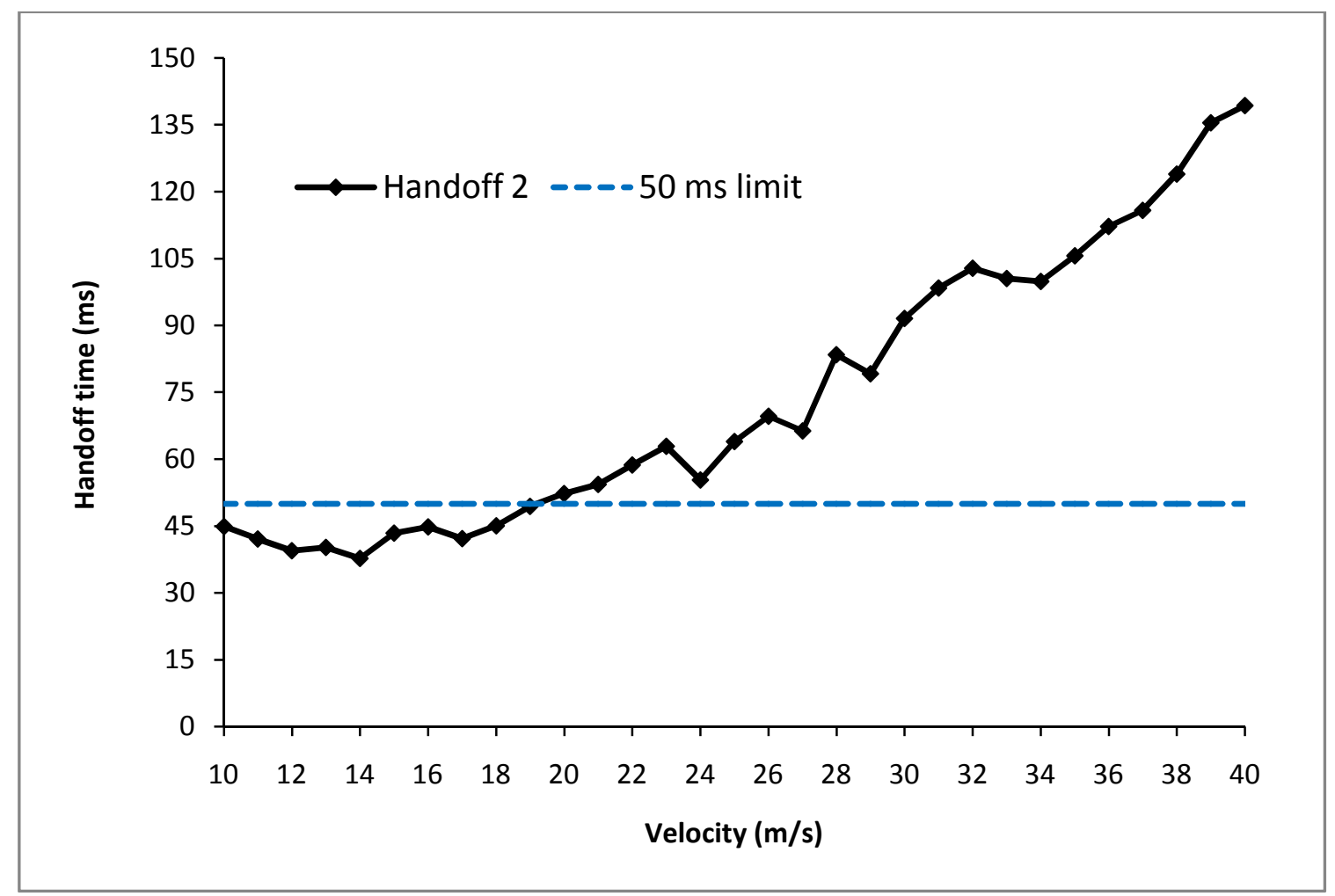

Figure 12. Simulated Handoff Times with Varied MS Speeds for Second Handoff

\section{CONCLUSION}

In this paper the performance of the handoff process in Mobile WiMAX network was investigated. Simulation was done with the NS-2 simulator. As NS-2 does not have all of the necessary components to support the implementation of Mobile WiMAX, two additional modules known as mobility and WiMAX from NIST are used in parallel with it. The parameters of the WiMAX module were adjusted and investigated to achieve shorter handoff duration time during both handoffs. After the adjustment of all the parameters, the velocity of the MS was varied within the range from $10 \mathrm{~m} / \mathrm{s}$ to $40 \mathrm{~m} / \mathrm{s}$. The main objective of this research was to find out the parameters that have the greatest impact on handoff performance as well as handoff latency during handoff in Mobile WiMAX. We have seen that some of the parameters have a direct influence on the handoff latency while others do not.

\section{REFERENCES}

[1] "IEEE Standard for Information technology, Telecommunications and information exchange between systems, Local and metropolitan area networks, Specific requirements", <url: http://standards.ieee.org/getieee802/download/802.11-2007.pdf>, retrieved December 2010.

[2] Encyclopedia Britannica, "WiMAX," <url: http://www.britannica.com/EBchecked/topic/1017801/WiMax >, retrieved December 2010.

[3] Zhang, Y., "Vertical Hando_ between 802.11 and 802.16 Wireless Access Networks," Waterloo, Canada, pp. 3, 2008. 
International Journal of Distributed and Parallel Systems (IJDPS) Vol.3, No.5, September 2012

[4] Diab, B. W.,Tohme, S., "End-to-End Security and Seamless Handover Solution for Real-Time Communications over 3G Networks," ACM, New York, pp.1, 2009.

[5] Pooja, B., Bijender M., "Analysis of Handover in Wimax for Ubiquitous connectivity", International Journal of Computational Engineering Research, ISSN: 2250-3005, Vol. 2, July-August 2012.

[6] Makelainen, A., "Analysis of Handoff Performance in Mobile WiMAX,” pp.12-68, 2007.

[7] WiMAX Forum: Feb 2006. "Mobile WiMAX - Part I: A Technical Overview and Performance Evaluation,"

url:<http://www.wimaxforum.org/technology/downloads/Mobile_WiMAX_Part1_Overview_and_Perfor mance.pdf $>$ retrieved January 2011.

[8] NIST Information Technology Laboratory "Seamless and Mobility," < url:

http://www.nist.gov/itl/antd/emntg/ssm_seamlessandsecure.cfm>, retrieved December 2010.

[9] ISO. "ISO/IEC 11172-1:1993 - Information technology -- Coding of moving pictures and associated audio for digital storage media at up to about 1,5 Mbit/s -- Part 1: Systems".<url: http://www.iso.org/iso/iso_catalogue/catalogue_tc/catalogue_detail.htm?csnumber=19180> Retrieved 2011-01-10. 